\title{
Weld Defect Detection Using Iterative Image Reconstruction Methods
}

\author{
Dr. B. Venkatraman ${ }^{1}$, M.M Anishin Raj2* and Dr. V. Vaithiyanathan ${ }^{3}$ \\ ${ }^{1}$ Assoc:Director, Radiological Safety and Environmental Group, IGCAR, Kalpakkam-603102, \\ India; bvenkat@igcar.gov.in \\ 2 Asst Prof., Department of ICT, SASTRA University, Thanjavur-613401, India; anishinraj@gmail.com \\ ${ }^{3}$ Assoc Dean Research, School of Computing, SASTRA University, Thanjavur-613401, India; vvn@it.sastra.edu
}

\begin{abstract}
Non Destructive Evaluation (NDE) is a technique to detect the defects without affecting the intended application or the structure, which is mainly used for damage assessment and quality control. NDE is basically used for automation of defect quantification. This paper presents a method for weld defect detection using iterative method for image reconstruction. Image reconstruction is a technique of reconstructing the image of object from the radon transform of the object, where radon transform is obtained by passing an energy source through the object in various angles and collecting the projected data on a detector. Weld defects such as slag inclusion, lack of penetration, and burn through can cause serious disaster if it is undetected at the time of weld process. The system of linear equation under consideration in Computed Tomography (CT) are basically very large in size, so the direct method takes too much of computational time which makes it impractical for implementation. Cimmino's method and Simultaneous Algebraic Reconstruction Technique (SART) are iterative methodologies for image reconstruction which are best suited for solving large scale linear system where direct method fails due to very large time consumption. This paper compares the experimental results of image reconstruction of weld defect radiographic images using SART and Cimmino's method for various test images with varying number of projections.
\end{abstract}

Keywords: Cimmino's Method, SART, Radon Transform, Tomography.

\section{Introduction}

Image processing has an important role in NDE for sizing of defect and image analysis. The various types of NDE techniques are tomography, infrared thermography, radiography, acoustic emission testing, and ultrasonic testing etc, where this paper focuses on tomography. Image reconstruction is a methodology through which, the image of an interior part of an object is reconstructed from the projection data of the object. The most effective type of reconstruction technique is the one which is able to reconstruct good quality CT images even when the projected data are highly noisy. Through CT we can detect the internal defects without damaging the structure by reconstructing a $2 \mathrm{D}$ or $3 \mathrm{D}$ image of the cross-section of the defective region [1].

CT has very high impact on medical field, where image of damaged parts of human are being reconstructed for disease diagnostic. Here, in this paper we are concentrating on image reconstruction of weld defect radiographic simulated images rather than on medical or other fields.

The burn through weld defect occurs when surplus of weld metal pierce into the weld zone, but slag Inclusion weld defect occurs when non metallic material get tangled in between base metal and weld metal. Lack of Penetration weld defect occurs due to the insufficiency of penetration of

* Corresponding author:

M. M. Anishin Raj (anishinraj@gmail.com) 
the weld metal into the joint. These weld defects can make serious disaster in boilers or chemical factories etc, since it is immovable and can't be identified once installed.

The quality of the density distribution of the cross section of the object reconstructed from the projected data using the iterative methodology or filtered back projection methodology depends on various parameter such as number of projection, type of iterative methodology used for reconstruction, projection data acquired etc. In iterative technology the cross section image is considered as an array of unknown values which are being represented as an algebraic equation in terms of projected data.

So many research works are being performed in the field of Simultaneous Algebraic Reconstruction Technique (SART), which is an enhancement of ART algorithm proposed by great scientist Kaczmarz. Tiejun Xu et.al used ART algorithm to estimate the current distribution which is a different application of iterative algorithm, rather than image reconstruction [2]. Weld defect detection such as Lack of Penetration and Porosity is done using Computed tomography by $\mathrm{M}$. Jovanovic et.al for sample weld images [3]. Bao-dong Liu et.al used SART and OSEM method for image reconstruction for long cylindrical object like pipe[4]. Y. J Roh and H. S Cho has implemented three dimensional image reconstruction using uniform and simultaneous algebraic reconstruction [5].

Carlos et.al developed a methodology for parallelization of block iterative Cimmino algorithm in which the data distribution and parallelism is handled by MUMPS (multi-frontal sparse solver) [6]. Stefania et.al proposed an extension of Cimmino's reflections algorithm to inconsistent least squares problems. The work presented two extensions and a corresponding convergence analysis of the classical Cimmino algorithm for iteratively computing constrained solutions to inconsistent least-squares solutions [7].

Gianfranco Cimmino, the distinguished Italian mathematician found out an iterative method for solving large linear system, where direct method like Gauss-Jordan, Gauss elimination and Crout's method will not work due to very large time consumption $[8,9]$. Gauss elimination method is a direct method where the system of linear equations is transformed to an upper triangular system which in turn increases computation time. Gauss-Jordan method is a modification of Gauss elimination method where the system of linear equation is brought down to a diagonal matrix. The Cimmino method is highly used in CT which is a combination of radiation technology and digital image processing principles applied in the field of medical diagnostics, astronomy and geography. Nowadays, it is widely used in other application areas such as astronomy and parallel computing, since it can be executed in parallel in multiple processors thereby enhancing the efficiency.

Gauss-Jacobi and Gauss-Siedal are iterative process with successive approximations which are highly suitable for computer operations [10]. But, the process of iteration will converge only if in each equation of the system, the coefficient of diagonal entries of the matrix is larger in magnitude than the sum of the magnitude of the other coefficients in the row. This condition of diagonally dominance is not satisfied in CT.

This paper compares two iterative reconstruction techniques such as Cimmino's and SART methods for various test images for quantification of the quality of reconstructed images for numerous numbers of projections.

\section{Cimmino's and SART Image Reconstruction Methods}

There are varioustype of algebraic reconstruction techniques like Multiplicative Algebraic Reconstruction Technique (MART), Improved Iterative Algorithm for Sparse Objects (IIASOR), Adaptive Algebraic Reconstruction Technique (AART), etc [11]. But, in this paper we have used SART and Cimmino's method for the reconstruction of simulated weld defect images. SART is an improvement of the ART for quick convergence. The equation for iterative reconstruction of unknown using SART can be represented as below [12].

$$
y_{j}^{v+\mathbf{1}}=y_{j}^{v}+\frac{\lambda}{T_{+, j}} \sum_{i=1}^{M} \frac{T_{i, j}}{T_{i,+}}\left(b_{1} i-T_{\mathbf{1}}(i,:) \cdot\left(y^{\mathbf{\top}}((v))\right)\right)
$$

Where, ' $y$ ' is the voxel's values, $\lambda$ is the relaxation factor, ' $v$ ' is the correction step, ' $b$ ' is the projection data, ' $T$ ' is the weight matrix where $j=1: N$. In the above equation, $\mathrm{Ti},+$ and $\mathrm{T}+, \mathrm{j}$ can be expressed as:

$$
\begin{aligned}
T_{i,+} & =\sum_{j=1}^{N} T_{i, j} \text { where, } \mathrm{i}=1: \mathrm{M} \\
T_{+, j} & =\sum_{i=1}^{M} T_{i, j} \text { where, } \mathrm{j}=1: \mathrm{N}
\end{aligned}
$$

Consider $A x=b$, as a system of linear algebraic equation, where $A$ is the matrix of size $n \times n$ and $x$ is the matrix to be 
reconstructed and 'b' being the projected data, the point $\mathrm{xi}^{(0)}$ which is a reflection of initial point $\mathrm{x}^{(0)}$ with respect to the hyper plane can be represented as:

$$
x_{i}^{(0)}=x^{(0)}+2 \frac{b_{i}-<a_{i}, x^{(0)}>}{\left\|a_{i}\right\|^{2}}
$$

The Cimmino's equation is based on the fact that all the reflection of the initial point $\mathrm{x}^{(0)}$ with respect to the ' $\mathrm{n}$ ' hyper plane lies on a hyper sphere, where the common point of ' $n$ ' hyper plane form the center of the hyper sphere which is the solution for the system of linear equation under consideration [7][8].

The Cimmino's equation can be represented in matrix form as shown below:

$$
\left.x_{j}^{v+1}=x_{j}^{v}+\frac{\lambda}{M} \sum_{i=1}^{M} \mathbb{\llbracket} \frac{T_{i j}}{\left\|T_{i, i}\right\|^{2}\left(b_{i}-T_{i,:} \mathbb{L}\right.} \cdot x^{v}\right)
$$

Where, ' $x$ ' being the voxel values, $\lambda$ the relaxation factor, ' $v$ ' is the correction step, ' $\mathrm{b}$ ' is the projection data, and ' $\mathrm{T}$ ' is the weight matrix where $\mathrm{j}=1 \mathrm{~N}$.

\section{Results and Discussion}

Various weld defects used to occur during welding process, in which few are burn through, cold lap, porosity, cluster porosity, lack of penetration, incomplete fusion, root undercut etc. But, in this paper we are reconstructing the image of burn through, slag inclusion and lack of penetration weld defect only. Figure 1 shows the simulated weld defects such as burn through, slag inclusion and lack of penetration. Figure 2 shows the binarized lack of penetration weld defect animated image. Figure 3 and Figure 4 shows the reconstructed images using Cimmino's method and SART method respectively for 3, 16 and 32 numbers of projections for the weld defect image. The reconstructed image of binarized lack of penetration weld defect, which occurs when the weld metal fall short to penetrate the joint, is shown in Figure 3 and Figure 4.

The results of reconstruction using Cimmino's method and SART for lack of penetration weld defect are being shown in Figure 3 and Figure 4. From the figures it is clear that the accuracy of reconstruction for different number of projections $(3,16,32)$ are higher for Cimmino's method

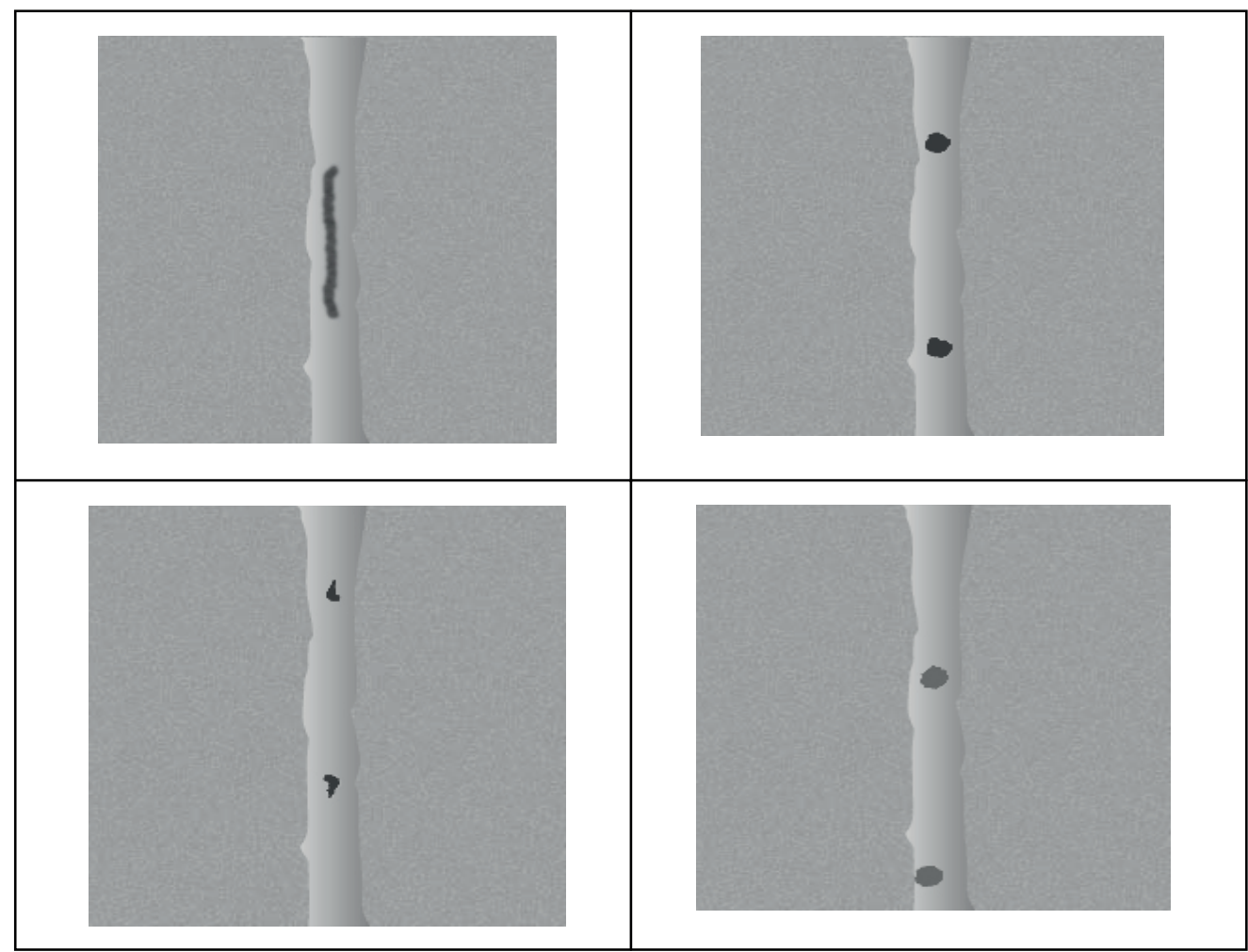

Figure 1. Simulated (Animated) input test images (lack of penetration, slag inclusion, burn through) weld defects. 


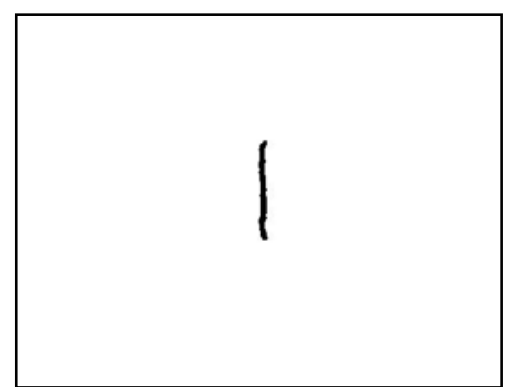

Figure 2. Binarized input image of lack of penetration.
Table 1. Performance of reconstruction using Cimmino's method

\begin{tabular}{lccc}
\hline $\begin{array}{l}\text { Performance } \\
\text { Parameters }\end{array}$ & \multicolumn{3}{c}{ Cimmino's Method } \\
\cline { 2 - 4 } & 3 projections & 16 projections & 32 projections \\
\hline $\begin{array}{l}\text { Mean absolute } \\
\text { Error }\end{array}$ & $2.66 \mathrm{e}-006$ & $9.395 \mathrm{e}-008$ & $2.2413 \mathrm{e}-008$ \\
$\begin{array}{l}\text { Correlation } \\
\text { Coefficient }\end{array}$ & 1 & & 1 \\
Elapsed Time(sec) & 8.714195 & 22.453043 & 36.805 \\
\hline
\end{tabular}

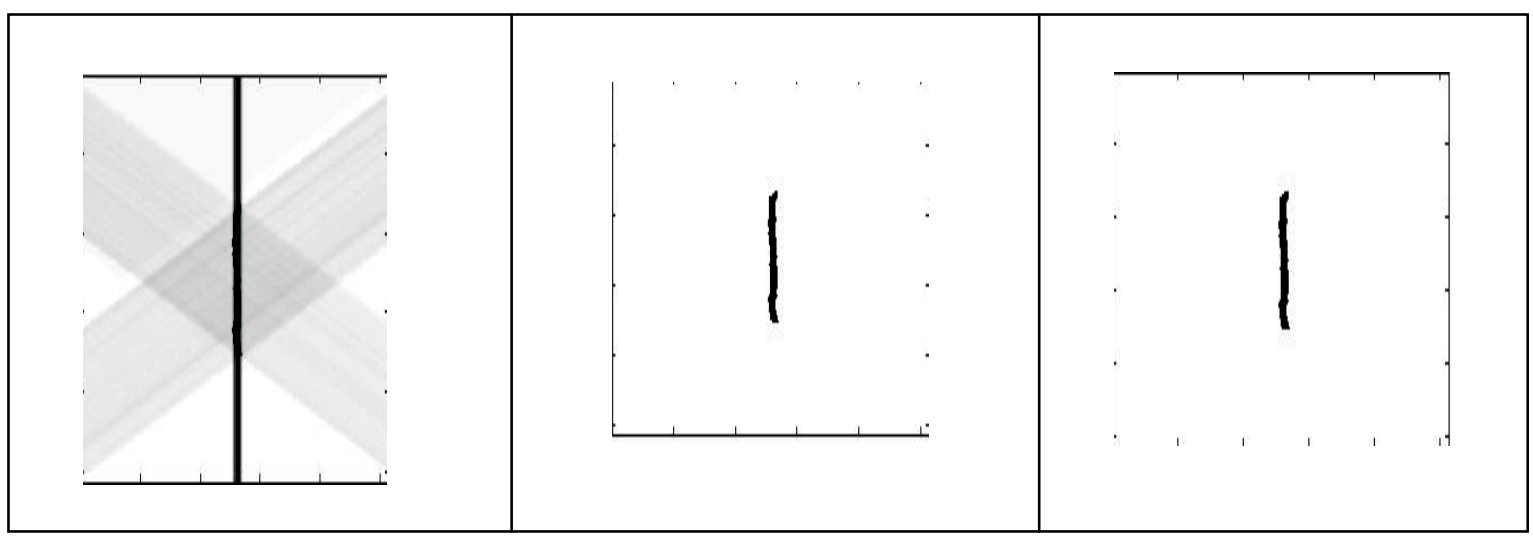

Figure 3. Results of reconstruction using Cimmino's method for 3, 16 and 32 numbers of projections of the binarized input image of lack of penetration.

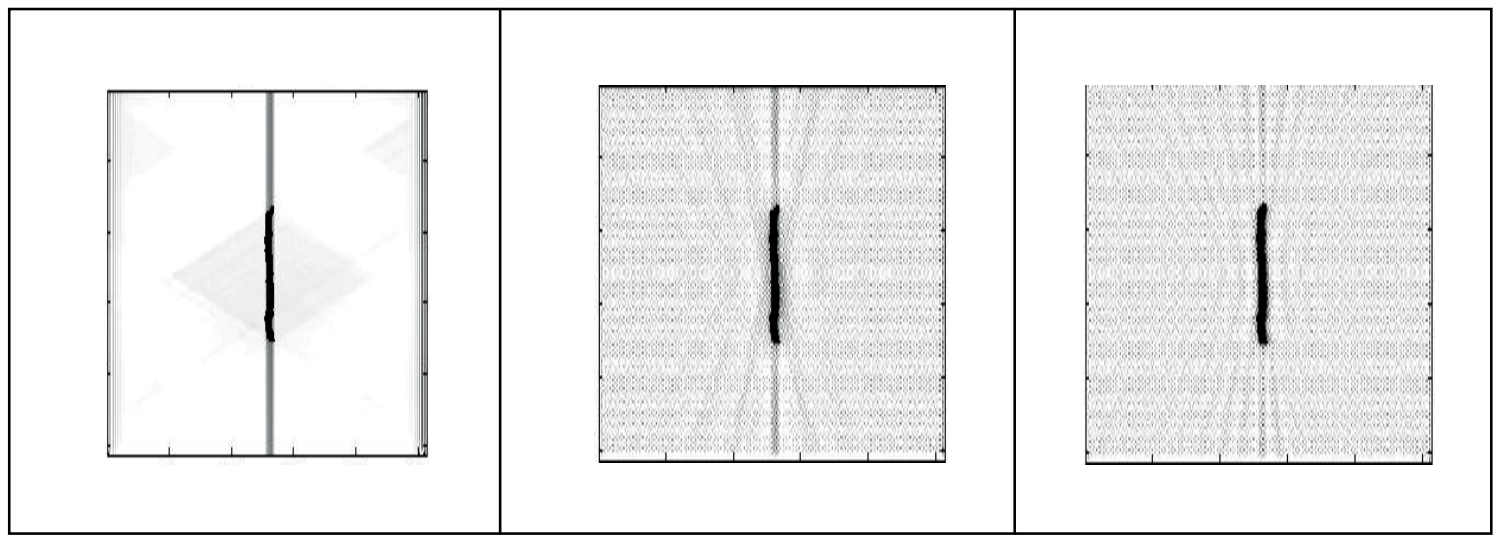

Figure 4. Results of reconstruction using SART for 3,16 and 32 numbers of projections of the binarized input image of lack of penetration.

when compared to SART. The Mean Absolute error, correlation coefficient and elapsed time are being depicted in the Table 1 and Table 2. From the Table 1 and Table 2 it is clear that the correlation coefficient is higher and Mean absolute error is lower for Cimmino's method. The graphical representation of performance shown in table is depicted in the Figure 5.

\section{Conclusion}

Weld defects such as lack of penetration, burn-through and slag inclusion are detected using iterative image reconstruction method such as Cimmino's method and SART method. The weld defect undetected in initial stage can lead to large disaster particularly in chemical factories 
Table 2. Performance of reconstruction using SART

\begin{tabular}{lccc}
\hline Performance Parameters & \multicolumn{2}{c}{ SART } \\
\cline { 2 - 4 } & 3 projections & 16 projections & 32 projections \\
\hline Mean absolute Error & 0.0435 & 0.1439 & 0.1328 \\
Correlation & 0.6198 & 0.4369 & 0.4678 \\
Coefficient & 1.988 & 3.4473 & 9.3134 \\
Elapsed Time(sec) &
\end{tabular}

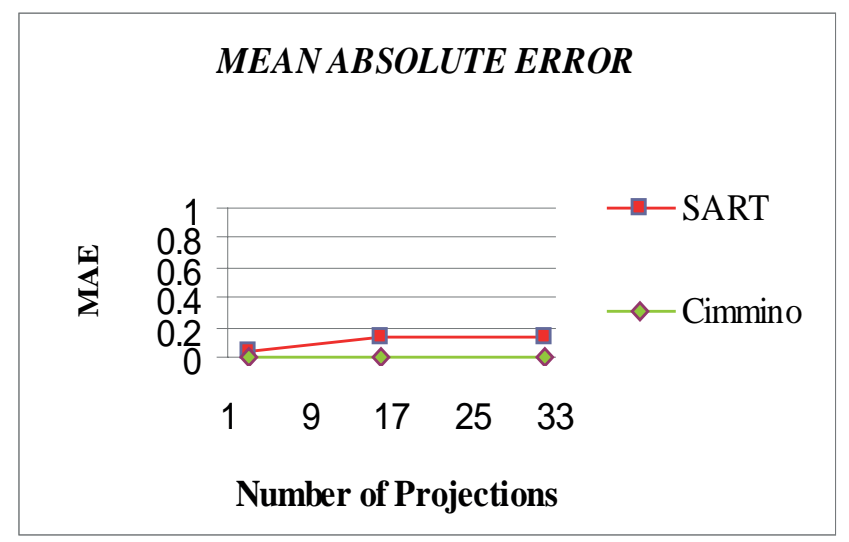

(a)

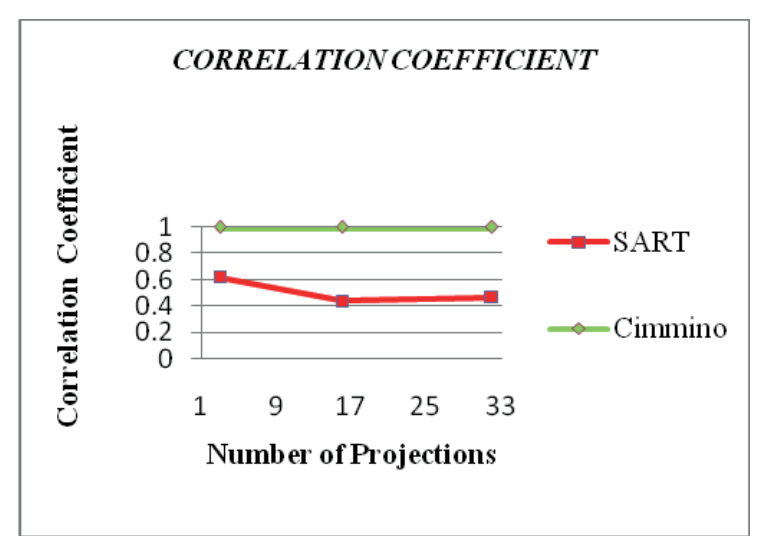

(b)

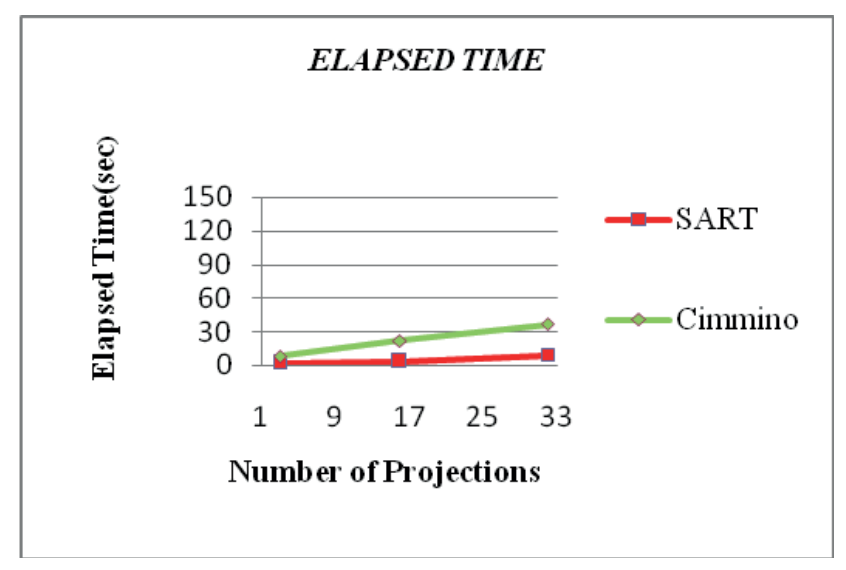

(c)

Figure 5. Graphical representation of performance of reconstruction using Cimmino's method and SART for the values shown in table 1 and table 2: (a) Mean absolute Error (b) Correlation Coefficient (c) Elapsed Time.

etc. The results show that the reconstruction by Cimmino's method is providing better correlation coefficient and lesser Mean Absolute error when compared to Simultaneous Algebraic Reconstruction Technique (SART). Weld defect detection in radiographic images by Cimmino's method of iterative image reconstruction technique provides a good result.

\section{References}

1. Kak C and Slaney M (1988). Principles of Computerized Tomographic Imaging, The Institute of Electrical and electronics Engineers, Inc., IEEE Press, New york.

2. Xu T, Rong M Z et al. (2009). The estimation of the current density distribution in a moving arc root using the ART 
algorithm,.IEEE Transactions on Plasma Science, Vol 37(7), 1311-1317.

3. Jovanovic M, Kosec L et al. (2012). Examination of weld defects by computed tomography, Metalurgija, vol 51(2), 233-236.

4. Liu B, Zeng L et al. (2008). Algebraic reconstruction technique class for linear scan CT of long object, Proceedings of the 17th World Conference on Nondestructive Testing, Shanghai, China.

5. Roh Y J, Kim B M et al. (2001). Three dimensional volume reconstruction of an object from $\mathrm{X}$-ray image, Proceedings of SPIE Vol 4190, 181-191.

6. Balsa C, Guivarch R et al. (2010). An hybrid approach for the parallelization of a block iterative algorithm, Proceedings of 9th International Conference of High Performance Computing for Computational Science (VECPAR'10), Berkeley, USA.

7. Petra S, Popa C et al. (2008). Extended and constrained Cimmino-type algorithms with applications in tomographic image reconstruction, Open Journal Systems, Heidelberg, 1-40.
8. Benzi M. (2004). Gianfranco Cimmino's contributions to numerical mathematics, Department of mathematics and computer science, Emory University, USA, 1-17.

9. Van Hemelryck Tessa, Wuyts S et al. (2007). The Implementation of Iterative Reconstruction Algorithms in MATLAB. Master's thesis, Department of industrial sciences and technology, University college of Antwerp, Belgium.

10. Milaszewicz J P (1987). Improving jacobi and gauss-seidel iterations, Journal of Linear Algebra and its Applications, Vol 93, 161-170.

11. Atkinson $\mathrm{CH}$ and Soria J (2007). Algebraic reconstruction techniques for tomographic particle image velocimetry, Proceedings of 16th Australasian Fluid Mechanics Conference Crown Plaza, Gold Coast, Australia.

12. Mueller K and Yagel R (2000). Rapid 3-D cone-beam reconstruction with the Simultaneous Algebraic Reconstruction Technique (SART) using 2-D texture mapping hardware, IEEE Transactions on Medical Imaging, Vol 19(12), 1227-1237. 